\title{
Fully polarimetric analysis of weather radar signatures
}

\author{
Michele Galletti*, David H. O. Bebbington, Madhu Chandra and Thomas Boerner \\ * German Aerospace Agency, Microwaves and Radar Institute, Radar Concepts Department \\ Oberpfaffenhofen, 82234 Wessling, Bayern, Deutschland \\ phone: + (49) 8153 283317, fax: + (49) 8153 281449, email: michele.galletti@dlr.de
}

\begin{abstract}
In this work the concept of depolarization response, namely the degree of polarization as a function of transmit polarization state, is investigated. Application examples are shown in the field of radar meteorology, namely for hydrometeor identification with fully polarimetric weather radar signatures. Data are from POLDIRAD, DLR research weather radar.
\end{abstract}

Index Terms - depolarization response, entropy, Kennaugh matrix

\section{INTRODUCTION}

A fully polarimetric radar is able to transmit pulses whose polarization state is switched every pulse repetition time (PRT) and is set to simultaneously receive the co- and cross- polar components of the backscattered signal. The first meteorological radar designed to measure complete scattering matrices of weather targets was developed at DLR about twenty years ago and is known to the weather radar community as POLDIRAD, acronym for polarization diversity radar. For a detailed technical description of the system, we refer to [1]. To collect the data presented in this work, POLDIRAD was operated to switch between horizontal and vertical polarization states on transmit, and was set to coherently receive the copolar and cross-polar components of the backscattered signal. Ideally, all elements of a scattering matrix should be measured simultaneously. However, since the transmit polarizations must be emitted sequentially, the scattering matrix measured by a fully polarimetric weather radar is affected by both mean motion of the target and decorrelation due to random displacements of the single scatterers. Special signal processing procedures were implemented to solve this problem [2].

A variable available to dual polarization coherent radar systems is the degree of polarization, obtainable from Wolf's coherency matrix [3]. The sensitivity of the degree of polarization to incoherent targets is however dependent on the transmit polarization state. To investigate this dependence, we applied unitary transformations to fully polarimetric radar signatures in order to compute the degree of polarization for different transmit states, and defined the depolarization response function $\mathrm{p}(\chi, \psi)$ as the degree of polarization of the backscattered wave as a function of the transmit polarization state. Interpretation of the observed signatures is given in terms of canonical incoherent scatterers, synthesized as sums of Kennaugh matrices representing clouds of coherent scatterers.
For a detailed description of standard radar meteorological variables like reflectivity $(Z)$, differential reflectivity $\left(Z_{D R}\right)$ or differential phase $\left(\Phi_{\mathrm{DP}}\right)$ we refer to [4], for a review of target decomposition theorems in radar polarimetry we refer to [5].

Aim of this paper is to show the utility of considering the whole depolarization response pattern (the degree of polarization for different transmit states) to retrieve information about incoherent targets. The application example carried out in the following uses fully polarimetric weather radar data to identify different hydrometeor classes, but further applications could be envisioned also for other fields of radar polarimetry like, for example, concealed target detection in polarimetric SAR.

\section{THEORY}

\section{A. Degree of Polarization}

Measurements done with a dual polarization coherent receiver can be considered as samples of a random Jones vector of the form

$$
E(t)=\left[\begin{array}{l}
E_{1}(t) \\
E_{2}(t)
\end{array}\right]=\left[\begin{array}{l}
\left|E_{1}(t)\right| e^{i \varphi_{1}(t)} \\
\left|E_{2}(t)\right| e^{i \varphi_{2}(t)}
\end{array}\right]
$$

The covariance of a random Jones vector (Wolf's coherency matrix $\mathrm{J}$ ) and the degree of polarization $\mathrm{p}$ read

$$
\begin{aligned}
& \operatorname{Cov}(E)=J=\left\langle E \otimes E^{+}\right\rangle \\
& p=\sqrt{1-\frac{4 \operatorname{det}(J)}{(\operatorname{trace}(J))^{2}}}=\frac{\lambda_{1}-\lambda_{2}}{\lambda_{1}+\lambda_{2}}
\end{aligned}
$$

As the lambdas are Wolf's matrix eigenvalues, the degree of polarization $p$ is a basis invariant quantity and as such does not depend on the orthogonal pair of polarimetric channels chosen to sample the backscattered wave. For a coherent target, the return is totally polarized, regardless of the transmit polarization state. For an incoherent target, the degree of polarization of the backscattered wave does in general depend on the polarization state of the transmitted wave. Such a function can then be indicated with $\mathrm{p}(\chi, \psi)$, where the greek letters $\chi$ and $\psi$ refer to the ellipticity and orientation angle describing the polarization state of the transmitted wave, while $\mathrm{p}$ indicates the degree of polarization of the backscattered wave. Such a function can be plotted directly on the Poincare 
sphere or with the help of surface plots, and could be referred to as depolarization response. In the most general case, it can be proven that the depolarization response has 6 extrema: two minima, two maxima and two saddle points [6]. The corresponding degrees of polarization will be indicated with $\mathrm{p}_{\text {MIN }}, \mathrm{p}_{\mathrm{MAX}}, \mathrm{p}_{\mathrm{S}}$.

It is useful to relate the degree of polarization to other relevant polarimetric variables appeared in the literature. Scattering entropy was defined for the quad pol and dual pol case mainly for SAR applications [7, 8]. Dual polarization entropy is equivalent to the degree of polarization (except that the first is expressed on a logarithmic scale whereas the second on a linear scale) and is therefore also dependent on transmit polarization state: $\mathrm{H}_{2}(\chi, \psi)$. On the other hand, entropy $(\mathrm{H}$, quad-pol) is a basis-invariant scalar and contains similar information as the minimal degree of polarization $\mathrm{p}_{\mathrm{MIN}}$.

\section{B. Depolarization Response}

Mixtures of hydrometeors can be described as weighted sums of normalized Kennaugh matrices.

$$
K=\sum_{i} w_{i} K_{i}
$$

Given the scattering matrix $\mathrm{S}_{\mathrm{i}}$ representing a coherent scatterer (sphere, spheroid, horizontal dipole, vertical dipole, canted dipole, right helix, left helix and so on) the corresponding Kennaugh matrix $\mathrm{K}_{\mathrm{i}}$ representing a cloud of such scatterers can be constructed by means of (5).

$$
\begin{aligned}
& K_{i}=\frac{1}{2}\left[Q^{T}\left[S_{i} \otimes S_{i}^{*}\right] Q\right] \\
& Q=\left[\begin{array}{cccc}
1 & 1 & 0 & 0 \\
0 & 0 & 1 & -j \\
0 & 0 & 1 & +j \\
1 & -1 & 0 & 0
\end{array}\right]
\end{aligned}
$$

Such a theoretical framework will be used to synthesize Kennaugh matrices corresponding to different clouds of scatterers. This model is better suited for the analysis of frozen precipitation, where mixtures of horizontal and/or vertical and/or roundish and/or irregular and/or canted ice crystals may occur.

We start however with an example about rain. In order to obtain a qualitative pattern for the depolarization response, we neglect the drop size distribution and we construct the corresponding Kennaugh matrix as a bimodal distribution of a cloud of spheres plus a cloud of horizontally oriented spheroids with the following $\mathrm{S}$ matrices:

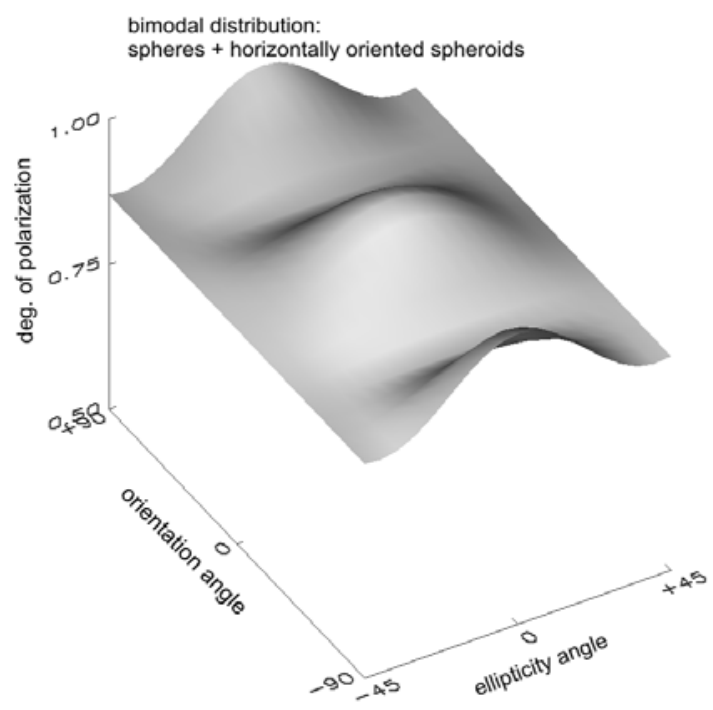

Figure 1. Depolarization Response of a bimodal distribution of horizontally aligned raindrops.

$S_{\text {sphere }}=\left[\begin{array}{ll}1 & 0 \\ 0 & 1\end{array}\right] \quad S_{\text {spheroid }}=\left[\begin{array}{cc}1 & 0 \\ 0 & 0.5\end{array}\right]$

$K=K_{\text {sphere }}+K_{\text {spheroid }}$

In this case the degree of polarization attains its minimal values on the circular/slant circle of the Poincare sphere, and its maximal values (1.0) at horizontal and vertical linear transmit (Fig. 1). It is worth noting that the presence of canted spheroids (Fig. $4 \mathrm{H}$ ) would induce a rotation (around the north-south axis of the Poincare sphere) of the minimal circle and of the maximal axis and, as a consequence, also canted raindrops are in principle detectable with an analysis of the depolarization response.

On the other hand, isotropic weather targets (characterized by differential reflectivity close to 0 ) have a different depolarization response pattern. Examples might be graupel, small hail, or randomly oriented dipoles.

A simple model for isotropic weather targets can be thought of as a cloud of randomly oriented spheroids. Considering Huynen parameters, a simple way to compute the degree of polarization as a function of the transmitted polarization state is obtained by considering the following Kennaugh matrix.

$$
\mathrm{K}_{\text {iso }}=\left[\begin{array}{cccc}
1+B_{0} & 0 & 0 & 0 \\
0 & 1 & 0 & 0 \\
0 & 0 & 1 & 0 \\
0 & 0 & 0 & -1+B_{0}
\end{array}\right]
$$




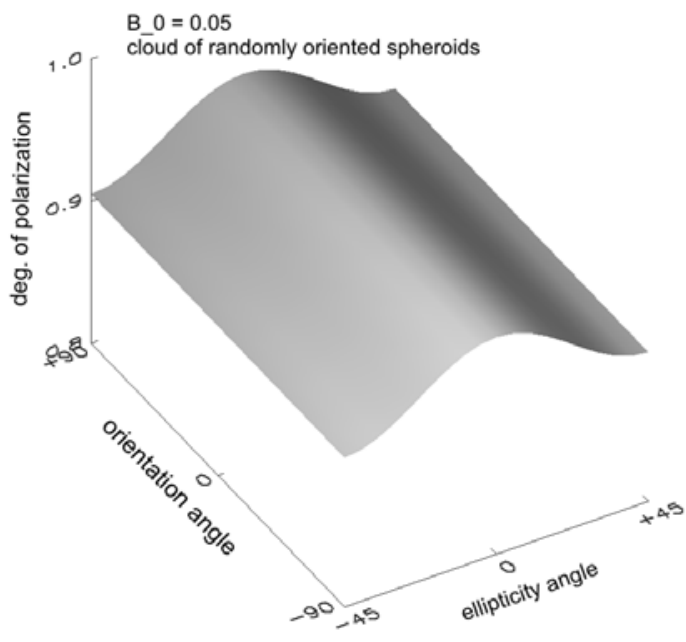

Figure 2. Depolarization Response for a cloud of randomly oriented, slightly oblate, spheroids $\left(\mathrm{B}_{0}=0.05\right)$

$\mathrm{B}_{0}$ ranges between 0 and 1 , depending if the spheroids are spheres $\left(\mathrm{B}_{0}=0\right)$ or dipoles $\left(\mathrm{B}_{0}=1\right)$. Simple algebra yields the following expression for the degree of polarization for a cloud of randomly oriented spheroids:

$$
p(\chi)=\frac{\sqrt{\cos ^{2}(2 \chi)+\left(1-B_{0}\right)^{2} \sin ^{2}(2 \chi)}}{1+B_{0}}
$$

Here, $\chi$ is the ellipticity angle and $\mathrm{B}_{0}$ is the generator of target structure. The above expression shows that, for an isotropic target, the degree of polarization attains its minimal values at the poles of the Poincare sphere and the maximal values at the equator (Fig. 2-3). Further, if we consider the quantity 1-p, the relation between the minimum and the maximum is a simple 3 $\mathrm{dB}$ difference.

$$
1-p_{\min }=\frac{2 B_{0}}{1+B_{0}} ; 1-p_{\max }=\frac{B_{0}}{1+B_{0}}
$$

The depolarization response of an isotropic target shows a number of symmetries, namely invariance with respect to orientation angle and handedness of the transmitted polarization state.

Before going into the analysis of experimental data, it is useful to give an overview on the behaviour of the depolarization response also in presence of more complex admixtures of incoherent scatterers. The graphs reported in the next page (Fig. 4, with letters from $\mathrm{A}$ to $\mathrm{H}$ ) were obtained from the numerical evaluation of equation (5), by choosing different combinations of Kennaugh matrices.

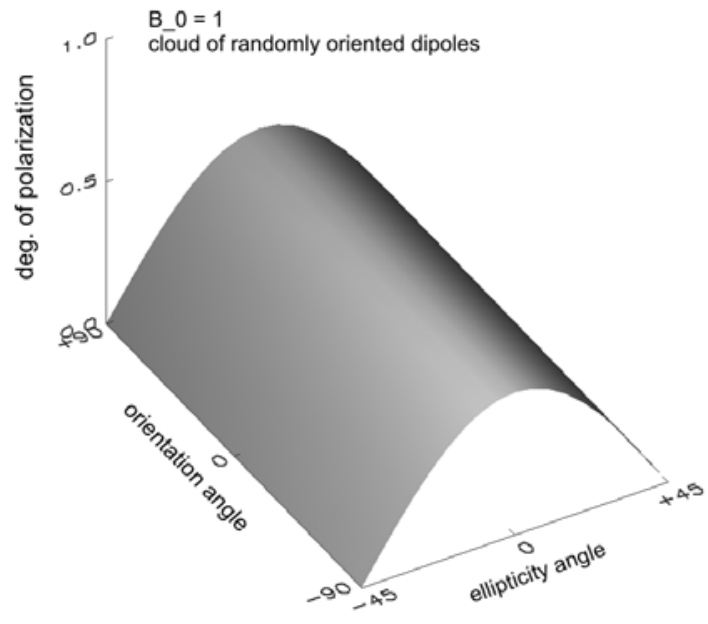

Figure 3. Depolarization Response for a cloud of randomly oriented dipoles $\left(\mathrm{B}_{0}=1\right)$

Fig. 4A is the depolarization response for a cloud of right-hand helices plus a cloud of left-hand helices. Fig. 4B was synthesized with the same kind of coherent scatterers but assigning a different weight to the two Kennaugh matrices coming in the sum. Even though we do not expect to find such scatterers in weather radar data, it is important to note how unbalanced weights of these two kinds of scatterers might break the depolarization response symmetry about the equatorial plane of the Poincare sphere. Scatterers with differential helicity can however be found in fully polarimetric SAR data.

Fig. 4C shows that for particular kinds of incoherent scatterers (in this case an admixture of horizontally-oriented and vertically oriented dipoles) the degree of polarization covers its whole dynamic range, from 0 to 1 , and it is indeed important to consider which polarization is being used for the interpretation of the signatures. Further, adding a cloud of spheres, for example, results in raising the minimal degree of polarization (Fig. 4D), whereas adding a cloud of randomly oriented dipoles would lower the maximal degree of polarization (figure not reported for compactness). The depolarization response for a cloud of horizontally oriented dipoles, plus a cloud of vertically oriented dipoles plus a cloud of spheres plus a cloud of randomly oriented dipoles yields a depolarization response as in Fig. 4E. The latter plot features a raise in the minimal degree of polarization and a lowering of the maximal degree of polarization with respect to the depolarization response reported in Fig. 4C. For weather radar investigations, it is important to note that the presence of irregularly shaped, roundish particles would affect the depolarization response in a way similar to the presence of a cloud of randomly oriented dipoles. So, Fig. 4E might also be interpreted as a cloud of $h$ dipoles plus a cloud of $\mathrm{v}$ dipoles plus a cloud of irregularly shaped spheres, thus excluding the physical presence of randomly oriented dipoles. 
A

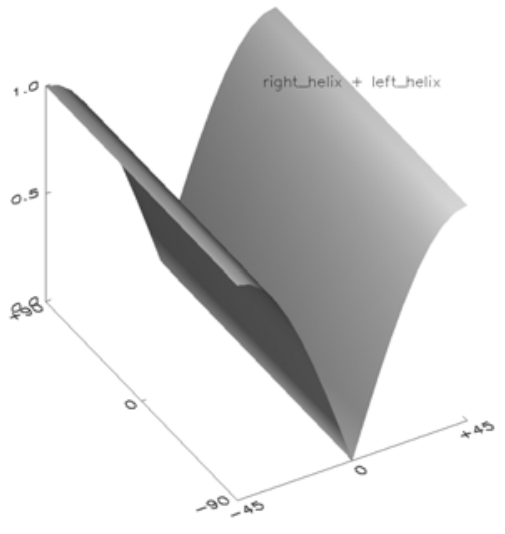

$\mathrm{C}$

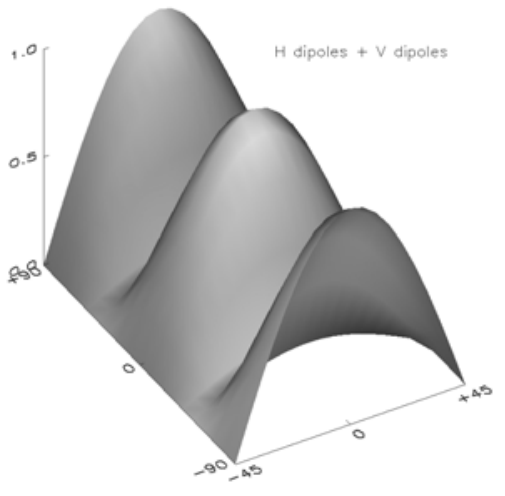

E

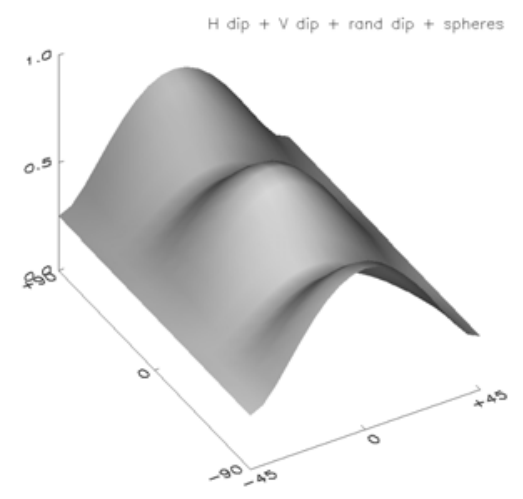

G

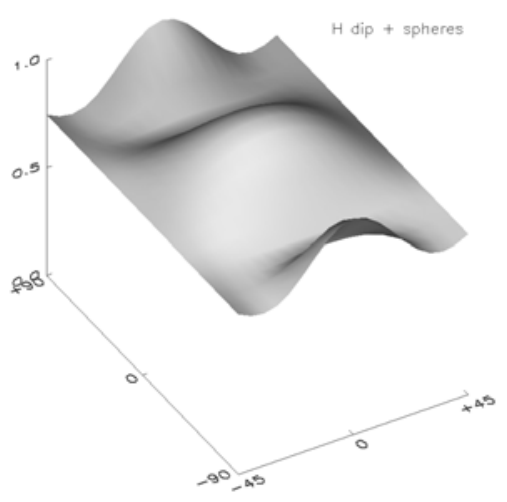

B

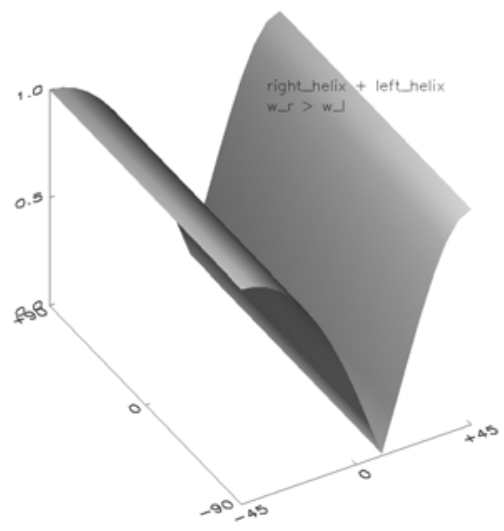

$\mathrm{D}$

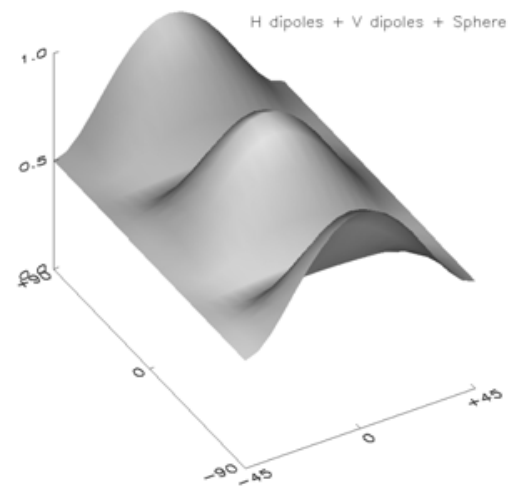

$\mathrm{F}$

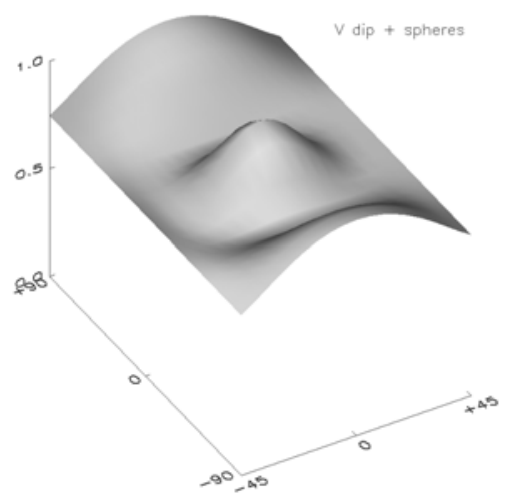

$\mathrm{H}$

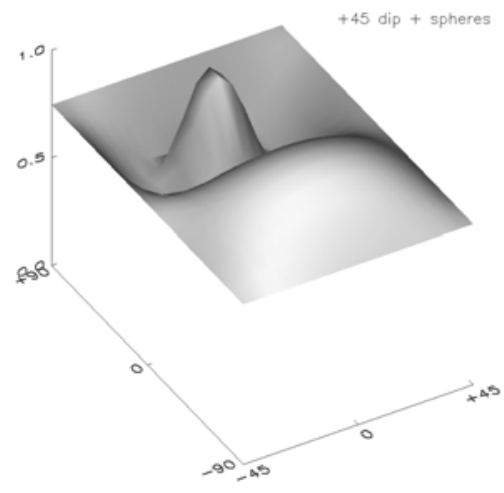

Figure 4. Depolarization Response for different admixtures of scatterers. Plots are labelled from A (top right) to $\mathrm{H}$ (bottom left). 

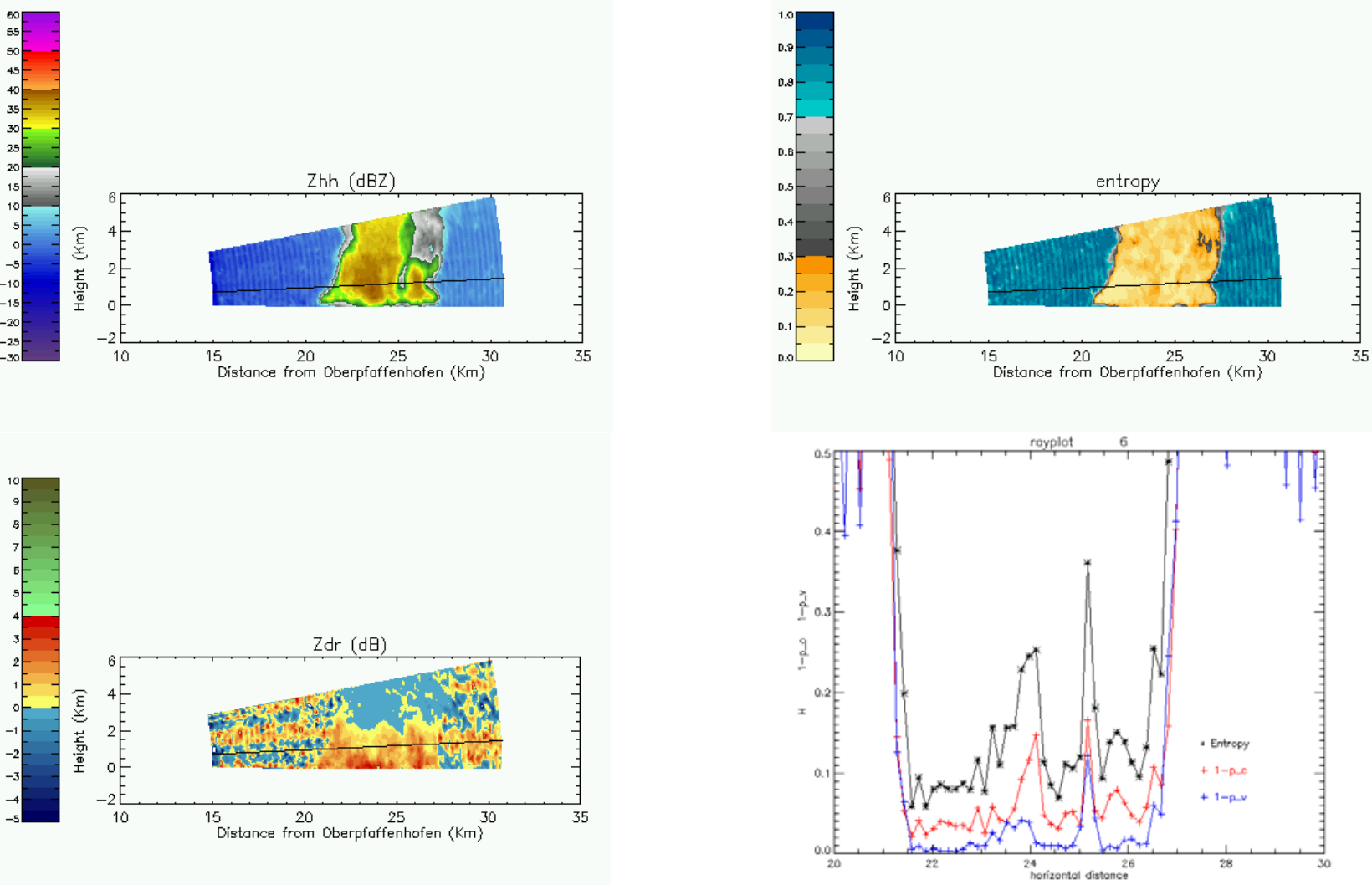

Figure 5. Convective event. Rayplot through rain and graupel.
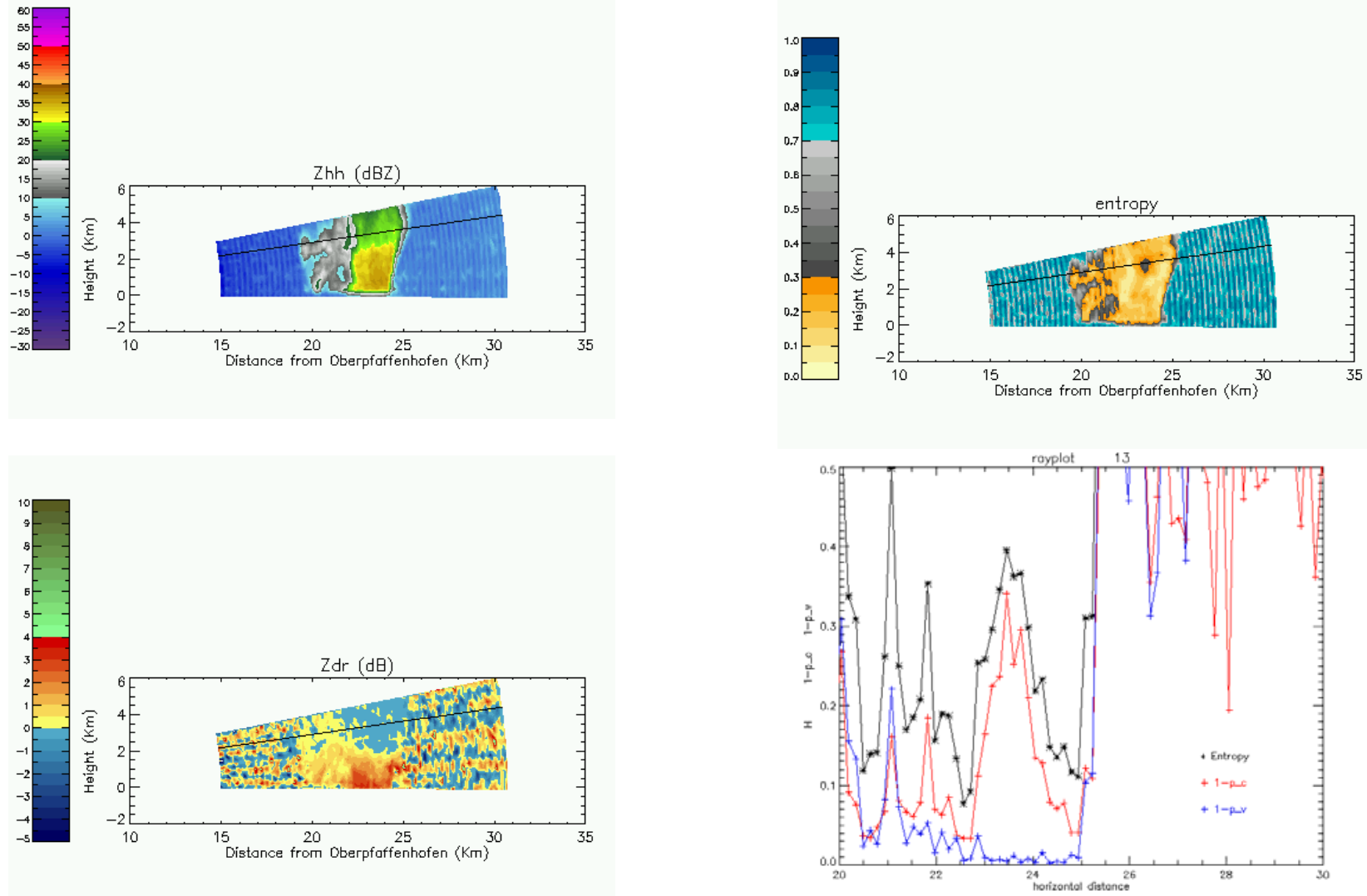

Figure 6. Convective event. Rayplot through ice crystals. 
An asymmetric weight of the oriented particles present in the illuminated volume would induce a differential amplitude of the lobes lying on the maximal degree of polarization axis. This effect is highlighted in Fig. 4F and Fig. 4G, representing the depolarization response of v-dipoles plus spheres and $\mathrm{h}$ dipoles plus spheres respectively.

Ultimately, as already stated above, the presence of canted spheroids (Fig. 4H) induces a rotation (around the north-south axis of the Poincare sphere) of the minimal circle and of the maximal axis. In Fig. $4 \mathrm{H}$ the two lobes on the maximal axis have different widths because only $+45^{\circ}$ oriented dipoles were included in the model. Adding $-45^{\circ}$ oriented dipoles would restore the same lobe width (figure not reported).

All these considerations suggest that, when fully polarimetric data are available, the depolarization response might bring valuable additional information. In the following we apply these principles to hydrometeor identification.

\section{DATA ANALYSIS}

Fig. 5 and Fig. 6 illustrate two RHIs for the same convective event. RHI stands for Range Height Indicator and is a scanning mode obtained by scanning in elevation with a fixed azimuth angle. The two RHIs (Fig. 5 and 6) are taken at slightly different azimuth angles. For each RHI, the ray in black in the reflectivity (upper left) entropy (upper right) and differential reflectivity (lower left) images corresponds to the rayplot shown in the lower right graph. For the data here considered, the degree of polarization at horizontal send $\left(\mathrm{p}_{\mathrm{H}}\right)$ behaves very similarly to the degree of polarization at vertical send $\left(\mathrm{p}_{\mathrm{V}}\right)$. The same happens for the pair $\mathrm{p}_{+45}$ and $\mathrm{p}_{-45}$, (henceforth indicated with $\mathrm{p}_{45}$ ) and for the pair $\mathrm{p}_{\mathrm{RHC}}$ and $\mathrm{p}_{\mathrm{LHC}}$ (henceforth indicated with $\mathrm{p}_{\mathrm{C}}$ ). Further, $\mathrm{p}_{45}$ behaves very similarly to $\mathrm{p}_{\mathrm{C}}$, and both variables generally take on larger values than $\mathrm{p}_{\mathrm{H}}$ or $\mathrm{p}_{\mathrm{V}}$. For compactness, only $\mathrm{p}_{\mathrm{V}}, \mathrm{p}_{\mathrm{C}}$ and $\mathrm{H}$ are reported on the graphs. In Fig. 5 a rayplot going through rain $(22-23 \mathrm{~km})$ and a mixture or rain and small hail $(24 \mathrm{~km})$ is chosen. As far as rain is concerned $(22-23 \mathrm{~km})$ the results are in qualitative accordance with the depolarization response graph for rain (Fig. 1) where $\mathrm{p}_{\mathrm{H}}$ and $\mathrm{p}_{\mathrm{V}}$ are almost 0 whereas $\mathrm{p}_{45}$ and $\mathrm{p}_{\mathrm{C}}$ take on larger values. A quantitative modeling for the values assumed by $p_{45}$ and $p_{C}$ must include, besides a more precise drop size distribution, the effects coming from Mie scattering at C-band. At $24 \mathrm{~km}$ distance from the radar a core of irregularly shaped hydrometeors (graupel, small-hail) is responsible for higher values of $\mathrm{H}, \mathrm{p}_{\mathrm{V}}$, and $\mathrm{p}_{\mathrm{C}}$. On the other hand, the ray shown in Fig. 6 goes through higher altitudes, where frozen hydrometeors are present. The ray was chosen for the peculiar feature present at 23-24 km from the radar: a core of hydrometeors with relatively high values of $\mathrm{H}$ and $\mathrm{p}_{\mathrm{C}}$ (above 0.3 ) and values of $p_{\mathrm{V}}$ close to 0 . This combination is unusual for snow or ice crystals, generally characterized by lower values of $\mathrm{H}$ and $\mathrm{p}_{\mathrm{C}}$ and $\mathrm{p}_{\mathrm{V}}$ values close to 0 .

In this case the analysis of degrees of polarization for different transmit states brings additional information.
We can in fact infer that the depolarization effects shown in Fig. 6 at 23-24 km do not come from a cloud of irregularly shaped ice crystals. This would in fact imply higher values also for $\mathrm{p}_{\mathrm{V}}$ that, in this case, keeps taking on values close to 0 . With reference to the depolarization response plots reported in Fig. 4, the observed signatures might be qualitatively interpreted as a mixture of horizontally oriented crystals, vertically oriented crystals plus spherical crystals.

$K=K_{\text {sphere }}+K_{H_{-} \text {dip }}+K_{V_{-} \text {dip }}$

A complete analysis of the depolarization response function allows assigning weights $\left(\mathrm{w}_{\mathrm{i}} \geq 0\right)$ to the different components of this model-based decomposition.

\section{CONCLUSIONS}

In this work, we investigated the dependence on transmit polarization state of the degree of polarization of a wave backscattered from an incoherent target. The concept of depolarization response is introduced to illustrate this dependence. Fully polarimetric data from POLDIRAD, DLR research weather radar, are used to substantiate the theory. It is found that the analysis of the whole depolarization response brings valuable additional information. A model-based decomposition based on the Kennaugh matrix is introduced for the purpose of hydrometeor identification. Experimental data were shown for the identification of ice crystals; potential applications could also be envisioned for canted hydrometeors.

\section{REFERENCES}

[1] A. Schroth, M. Chandra and P. F. Meischner, "A C-band coherent polarimetric radar for propagation and cloud physics research," Journal of Oceanic and Atmospheric Technology, Vol. 5, pp. 803-822, December 1988.

[2] V. Chandrasekar, J. Hubbert, V. N. Bringi and P. F. Meischner, "Interpolation procedures to construct complete polarimetric signatures of distributed targets," Proc. SPIE, 1748, pp. 200-212, 1992.

[3] M. Born and E. Wolf, Principles of Optics: Electromagnetic Theory of Propagation, Interference and Diffraction of Light, $7^{\text {th }}$ ed. Cambridge University Press, 1999.

[4] V. N. Bringi and V. Chandrasekhar, Polarimetric Doppler Weather Radar. Principles and applications, Cambridge University Press, 2001.

[5] S. R. Cloude and E. Pottier, "A Review of Target Decomposition Theorems in Radar Polarimetry," IEEE Transactions on Geoscience and Remote Sensing, vol. 34, no. 2, pp. 498-518, March 1996.

[6] D. H. O. Bebbington, "Fundamentals of Radar Polarimetry using Complex Spinor Formalism", a monograph under BAA \#99-025 for long range scientific projects

[7] S. R. Cloude and E. Pottier, "Concept of polarization entropy in optical scattering," Opt. Eng. 34, 1599-1610 (1995).

[8] S. R. Cloude, "The Dual Polarization Entropy/Alpha Decomposition: A PALSAR case study", Proceedings of POLINSAR 07 Workshop, ESAESRIN, 22-26 January 2007. 